Do small worlds synchronize fastest?

This article has been downloaded from IOPscience. Please scroll down to see the full text article.

2010 EPL 9048002

(http://iopscience.iop.org/0295-5075/90/4/48002)

View the table of contents for this issue, or go to the journal homepage for more

Download details:

IP Address: 134.76.162.165

The article was downloaded on 05/12/2012 at 08:11

Please note that terms and conditions apply. 


\title{
Do small worlds synchronize fastest?
}

\author{
C. Grabow ${ }^{1(a)}$, S. M. Hill ${ }^{2}$, S. Grosskinsky ${ }^{2}$ and M. Timme ${ }^{1,3}$ \\ ${ }^{1}$ Network Dynamics Group, Max Planck Institute for Dynamics and Self-Organization - 37073 Göttingen, \\ Germany, EU \\ ${ }^{2}$ Centre for Complexity Science, University of Warwick - Coventry CV4 7AL, UK, EU \\ ${ }^{3}$ Bernstein Center for Computational Neuroscience (BCCN) Göttingen - 37073 Göttingen, Germany, EU
}

received 4 March 2010; accepted in final form 11 May 2010

published online 15 June 2010

PACS 89.75.-k - Complex systems

PACS 05.45.Xt - Synchronization; coupled oscillators

PACS 87.19.1m - Synchronization in the nervous system

\begin{abstract}
Small-world networks interpolate between fully regular and fully random topologies and simultaneously exhibit large local clustering as well as short average path length. Smallworld topology has therefore been suggested to support network synchronization. Here we study the asymptotic speed of synchronization of coupled oscillators in dependence on the degree of randomness of their interaction topology in generalized Watts-Strogatz ensembles. We find that networks with fixed in-degree synchronize faster the more random they are, with small worlds just appearing as an intermediate case. For any generic network ensemble, if synchronization speed is at all extremal at intermediate randomness, it is slowest in the small-world regime. This phenomenon occurs for various types of oscillators, intrinsic dynamics and coupling schemes.
\end{abstract}

openácess Copyright (C) EPLA, 2010

Synchronization dominates the collective dynamics of many physical and biological systems $[1,2]$. It might be both advantageous and desired, for instance in secure communication [3], or detrimental and undesired, as during tremor in patients with Parkinson disease or during epileptic seizures $[4,5]$. Therefore, a broad area of research has emerged [6-8], determining under which conditions on the interaction strengths and topologies coupled units actually synchronize and when they do not. In a seminal work essentially founding the science of complex networks, Watts and Strogatz [9] suggested that a small-world topology of a network is particularly supportive of synchronization because small worlds exhibit high local clustering and simultaneously low average path length. Indeed, several detailed studies support this view by showing that at fixed coupling strength small-world networks tend to already synchronize at lower connectivity than many other classes of networks $[9,10]$; small worlds also more easily exhibit self-sustained activity [11].

These results suggest some key properties about the topological influence on the network synchronizability, i.e. the capability of a network to synchronize at all, but do not tell much about the speed of synchronization given that a network synchronizes in principle.

\footnotetext{
(a) E-mail: grabow@nld.ds.mpg.de
}

For any real system, however, it equally matters how fast the units synchronize or whether the network interactions fail to coordinate the units' dynamics on time scales relevant to the system's function (or dysfunction), cf. [12-15]. Yet this question is far from being understood and currently under active investigation [16-20]. In particular it is largely unknown how fast small worlds synchronize, an astounding fact given the seminal work on small-world networks [9] published more than a decade ago.

In this letter we study the speed of synchronization in generalized Watts-Strogatz ensembles and systematically compare the small-world regime to more regular and more random topologies. We find that small worlds synchronize faster than regular networks but still orders of magnitude slower than fully random networks. The observed increase of synchronization speed with randomness might be attributed $[9,17]$ to the simultaneous decrease of the average path length between two units in the network. We therefore compare ensembles of networks where the degree of randomness varies from completely regular to completely random such that the average path length stays constant. Here we find that networks synchronize slowest in the small-world regime. Within the entire model class, these results hold for any generic ensemble, i.e. synchronization speed may be intermediate or 
slowest but is never fastest in the small-world regime. This phenomenon occurs across many distinct systems, including phase oscillators, higher-dimensional periodic and chaotic systems coupled diffusively as well as neural circuits with inhibitory delayed pulse-coupling.

Consider $N$ Kuramoto oscillators [21] that interact on a directed network. The dynamics of phases $\theta_{i}(t) \in \mathbb{S}^{1}=$ $2 \pi \mathbb{R} / \mathbb{N}$ of oscillators $i$ with time $t$ satisfy

$$
\frac{\mathrm{d} \theta_{i}}{\mathrm{~d} t}=\omega+\sum_{j} J_{i j} \sin \left(\theta_{j}-\theta_{i}\right) \quad \text { for } i \in\{1, \ldots, N\},
$$

where $\omega$ is the natural frequency of the oscillators, $J_{i j}=$ $J / k$ is the coupling strength between two units and $k$ is the number of in-links to a unit. To analyze the purely topological impact on the synchronization times, we study the network dynamics in its simplest setting: we consider strongly connected networks ${ }^{1}$ with fixed in-degree $k$ and homogeneous total input coupling strengths such that full synchrony is achieved from sufficiently close initial conditions for all coupling strengths $J>0$ [20].

As the synchronous periodic orbit analyzed is isolated in state space, the relaxation time continuously changes with possible inhomogeneities, so the qualitative results obtained below are generic and also hold in the presence of small heterogeneities, cf. [22].

To systematically investigate the sychronization process in dependence of the topological randomness we first performed extensive numerical simulations of the collective dynamics. We start with regular ring networks where each unit receives directed links from its $k / 2$ nearest neighbors on both sides. Adapting the standard small-world model of Watts and Strogatz [9] to directed networks [23] we randomly cut the tail of each edge with probability $q$ and rewire it to a randomly selected node (avoiding double edges and self-loops). The small-world regime is characterized by a large clustering coefficient ${ }^{2}\langle C(q, k)\rangle$ and a small average path length ${ }^{3}\langle L(q, k)\rangle$. Here $\langle$.$\rangle denotes averaging$ over network realizations at given $q$ and $k$. To quantitatively fix the small-world regime we take

$$
\frac{\langle L(q, k)\rangle}{L(0, k)}<0.5 \text { and } \quad \frac{\langle C(q, k)\rangle}{C(0, k)}>0.85
$$

throughout this study. The results below are not sensitive to a change of these values. Starting each simulation from a random initial phase vector drawn from the uniform distribution on $[0, \pi)^{N}$ shows that synchronization becomes an exponential process after some short transients (fig. 1, inset), for all fractions $q \in(0,1]$ of randomness. Thus the distance

$$
d(t)=\max _{i, j} \operatorname{dist}\left(\theta_{i}(t), \theta_{j}(t)\right)
$$

\footnotetext{
${ }^{1} \mathrm{~A}$ network is strongly connected if there is a directed path between each ordered pair of nodes.

${ }^{2} C(q, k)$ denotes the actual divided by the possible number of directed triangles containing a given node $i$, averaged over all $i$.

${ }^{3} L(q, k)$ denotes the length of the shortest directed path between a given pair of nodes $(i, j)$, averaged over all $(i, j)$.
}

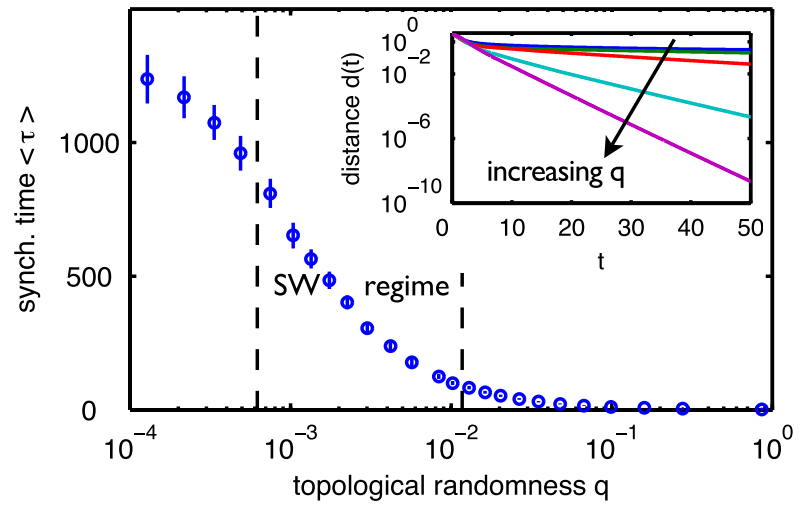

Fig. 1: (Color online) Synchronization times (4) monotonically decrease with randomness in network ensembles with fixed in-degree $k=20(N=1000$ nodes, $J=1$, averages $\langle\tau\rangle$ over 100 realizations of networks with random initial condition each; synchronization times ranging from $\langle\tau\rangle=1.3(q \rightarrow 1)$ to $\langle\tau\rangle=$ $1316(q \rightarrow 0)$; error bars give standard deviation). The smallworld regime (eq. (2), dashed vertical lines indicate bounds) appears not to be special at all. Inset: Distance $d$ to the synchronous state (3) decays exponentially with time $t$ after short transients for entire range of randomness $q \in[0,1]$; lines provide single realizations for $q \in\{0,0.008,0.04,0.2,1\}$.

from the synchronous state decays as

$$
d(t) \sim \exp (-t / \tau)
$$

in the long time limit, where $\operatorname{dist}\left(\theta, \theta^{\prime}\right)$ is the circular distance between the two phases $\theta$ and $\theta^{\prime}$ on $\mathbb{S}^{1}$.

The asymptotic synchronization time $\tau$ systematically depends on the network topology (fig. 1): regular ring networks $(q \rightarrow 0)$ are typically relatively slow to synchronize. Increasing randomness $q$ towards the small-world regime induces shorter and shorter network synchronization times, with small worlds synchronizing a few times faster than regular rings. Further increasing the randomness $q$ induces even much faster synchronization, with fully random networks $(q \rightarrow 1)$ synchronizing fastest (two orders of magnitude faster than small worlds in our examples). Thus in network ensembles with fixed in-degree small worlds just occur intermediately during a monotonic increase of synchronization speed, but are not at all topologically optimal regarding their synchronization time.

This might be expected intuitively, also from studies about synchronizability $[9,10]$, and one is tempted to ascribe faster synchronization to a shorter average path length that results from increasing randomness.

We therefore first systematically studied the synchronization time for generalized Watts-Strogatz ensembles of networks, specified by a function $k(q)$, where the average path length $\langle L\rangle$ is fixed while the degree of randomness $q$ varies ${ }^{4}$.

We were surprised to find a non-monotonic variation of synchronization time with randomness (fig. 2). In

\footnotetext{
${ }^{4}$ We choose an appropriate in-degree $k(q)$ for each given randomness $q$ from numerically determined calibration curves such that $\langle L(q, k(q))\rangle$ is fixed.
} 


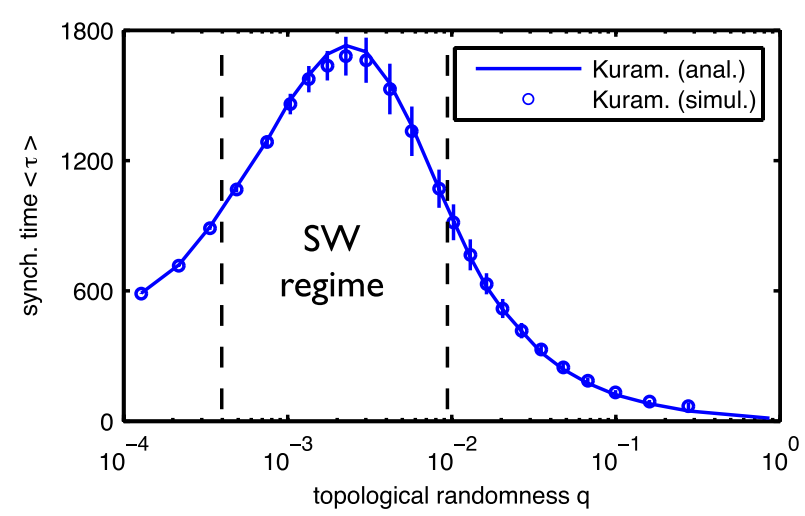

Fig. 2: (Color online) Small worlds exhibit slowest synchronization in network ensembles with fixed average path length. Here $N=1000$ and $\langle L\rangle=4$; small-world region (2) is located between dashed vertical lines.

particular, networks with intermediate randomness in the small-world regime synchronize slowest. Analytical calculations support this view. In the dynamics linearizing (1) close to the synchronous state (where $\theta_{i}(t) \equiv \theta_{j}(t)=: \theta(t)$ ) phase perturbations $\varphi_{i}(t):=\theta_{i}(t)-\theta(t)$ evolve according to

$$
\frac{\mathrm{d} \varphi_{i}}{\mathrm{~d} t}=\sum_{j} \Lambda_{i j} \varphi_{j}, \quad \text { for } i \in\{1, \ldots, N\} .
$$

Here the stability matrix $\Lambda$ coincides with the graph Laplacian defined as

$$
\Lambda_{i j}=J_{i j}\left(1-\delta_{i j}\right)-J \delta_{i j}
$$

and $\delta_{i j}$ is the Kronecker-delta. Close to every invariant trajectory the eigenvalue $\lambda_{2}$ of the stability matrix $\Lambda$ that is second largest in real part dominates the asymptotic decay; therefore, $\lambda_{2}$ here determines the asymptotic synchronization time via $\tau=-\frac{1}{\operatorname{Re} \lambda_{2}}$. This feature was recently shown to hold more generally for network systems where the stability matrix is not necessarily proportional to the graph Laplacian $[2,16,24]$.

Determining the eigenvalues of the stability matrices of networks with fixed average path lengths yields synchronization time estimates that well agree with those found from direct numerical simulations, cf. fig. 2. This independently confirms that synchronization is indeed slowest for small-world networks.

How does synchronization speed vary with randomness for more general ensembles $k(q)$ ? A systematic study of the synchronization time as a function of both in-degree $k$ and randomness $q$ (fig. 3 ) reveals an interesting nonlinear dependence. Firstly, it confirms that for all networks with fixed in-degree $k$ the synchronization time is monotonic in the randomness $q$ and the small-world regime at intermediate randomness is not specifically distinguished. Secondly, the two-dimensional function $\langle\tau(q, k)\rangle$ implies that ensembles of networks with fixed path lengths all exhibit a non-monotonic behavior of the synchronization

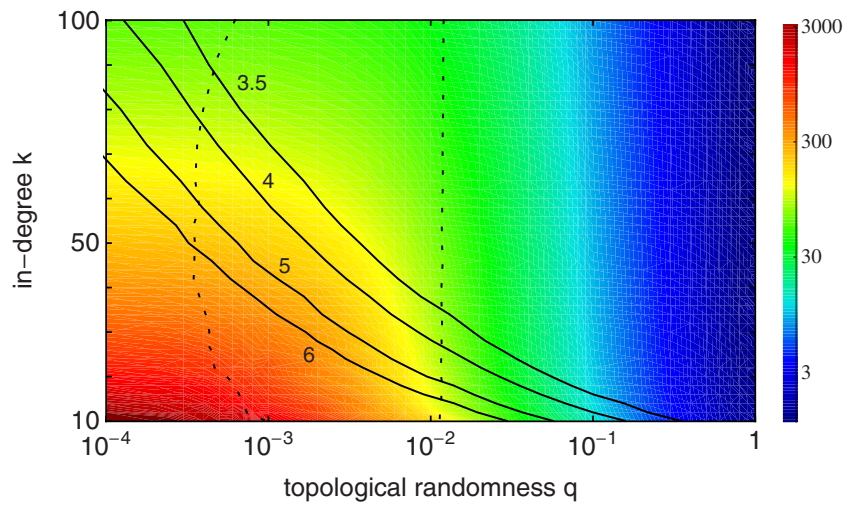

Fig. 3: (Color online) Nonlinear dependence of synchronization time on in-degree $k$ and topological randomness $q$ indicates that no generic ensemble $k(q)$ exhibits fastest synchronization in the small-world regime (logarithmic color scale ranging from $\langle\tau\rangle=4606$ (dark red) to $\langle\tau\rangle=1.1$ (dark blue)). Solid lines indicate ensembles of networks with fixed average path length from $\langle L\rangle=3.5$ (top) to $\langle L\rangle=6$ (bottom). The small-world regime (2) is located between the dashed lines.

time, with slowest synchronization for intermediate randomness.

Thirdly, considering graph ensembles characterized by any other smooth function $k(q), q \in[0,1]$, shows that this is a general phenomenon and the specific choice of an ensemble $k(q)$ is not essential.

In fact, as illustrated in fig. 3, for any generic network ensemble $k(q)$ (including ensembles with fixed in-degree and fixed path length as special choice) the synchronization speed $\langle\tau(q, k(q))\rangle$ is either intermediate or slowest, but never fastest at intermediate randomness, in particular in the small-world regime.

Is this phenomenon restricted to the specific class of Kuramoto oscillators? To answer this question, we explored the synchronization dynamics of various kinds of oscillators coupled in different ways, and consistently found qualitatively the same results. Specifically, in networks with fixed average path length, synchrony is consistently fast for regular rings, fastest for completely random networks, and slowest in the intermediate small-world regime (fig. 4).

For instance, we tested networks of diffusively coupled three-dimensional Rössler oscillators [1] satisfying

$$
\begin{aligned}
\dot{x}_{i} & =-y_{i}-z_{i}+\sum_{j} J_{i j}\left(x_{j}-x_{i}\right), \\
\dot{y}_{i} & =x_{i}+a y_{i}, \\
\dot{z}_{i} & =b+z_{i}\left(x_{i}-c\right),
\end{aligned}
$$

for $i \in\{1, \ldots, N\}$, where $J_{i j}=J / k$ define the diffusive coupling and the parameters $a, b$ and $c$ determine whether the oscillators are intrinsically periodic or intrinsically chaotic. The above phenomenon persists for both periodic and chaotic oscillators (fig. 4, triangles). 


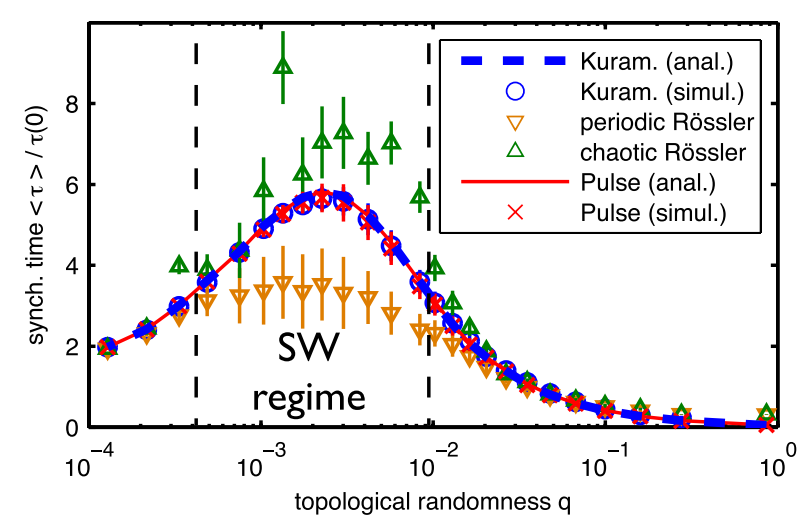

Fig. 4: (Color online) Synchronization is slowest in the small-world regime for various oscillator types and coupling schemes. Normalized average synchronization times $\langle\tau\rangle / \tau(0)$ vs. $q$ for Kuramoto, pulse-coupled and periodic as well as chaotic Rössler oscillators (network topologies as in fig. 2). Neurons with delayed pulse-coupling: $I=1.01, \gamma=1, J=$ $-0.2, \Delta=0.1$. Diffusively coupled Rössler oscillators: $a=0.2$, $c=5.7$; periodic oscillators: $b=1.7, J=2$; chaotic oscillators: $b=0.2, J=6$. The synchronization times are determined by measuring the distances $d=\max _{i, j}\left\{\left(\left(x_{i}-x_{j}\right)^{2}+\left(y_{i}-y_{j}\right)^{2}+\right.\right.$ $\left.\left.\left(z_{i}-z_{j}\right)^{2}\right)^{1 / 2}\right\}$ (Rössler), and $d=\max _{i}\left|\delta_{i}\right|$ (pulse-coupled) and fitting (4).

Moreover, we investigated the collective dynamics of pulse-coupled neural oscillators [14,25] with membrane potentials $V_{i}(t)$ satisfying

$$
\frac{\mathrm{d} V_{i}}{\mathrm{~d} t}=I-\gamma V_{i}+\sum_{j=1 ; j \neq i}^{N} \sum_{m \in \mathbb{Z}} J_{i j} \delta\left(t-\left(t_{j, m}+\Delta\right)\right) .
$$

Here, each potential $V_{j}$ relaxes towards $I>1$ and is reset to zero whenever it reaches a threshold at unity,

$$
V_{j}\left(t^{-}\right)=1 \Rightarrow V_{j}(t):=0, \quad t_{j, m}:=t, \quad \text { and } \quad m \mapsto m+1 .
$$

At these times $t_{j, m}$ the neuron sends a pulse that after a delay $\Delta>0$ changes the potential of post-synaptic neurons $i$ in an inhibitory (negative) manner. This neural system allows analytic computation [17] of an iterative map

$$
\delta_{i}(n T)=\sum_{j=1}^{N} A_{i j} \delta_{j}((n-1) T), \quad n \in \mathbb{N}
$$

for the perturbations $\delta_{i}(n T)$ of spike times close to the synchronous orbit of period $T=(1 / \gamma) \ln (1 /(1-\gamma / I))$. For homogeneous coupling $\left(J_{i j}=-J / k\right.$ for each existing connection) the elements of the stability matrix $A$ are given by $A_{i j}=A_{+}=(\gamma J) /\left(k\left(I e^{-\gamma \Delta}+\gamma J\right)\right)$ if there is a connection from $j$ to $i \neq j, A_{i i}=1-k A_{+}$for the diagonal elements and $A_{i j}=0$ otherwise, cf. [17]. As for the Kuramoto system, the prediction of synchronization times based on the eigenvalues of the matrix $A$ well agrees with those obtained from direct numerical simulations (fig. 4, crosses and solid line).
These results confirm that, largely insensitive to the type of oscillators (phase, multi-dimensional, neural), their intrinsic dynamics (periodic, chaotic) and their coupling schemes (phase-difference, diffusive, pulse-like), networks with fixed average path length consistently synchronize slowest in the small-world regime at intermediate randomness. Further numerical analysis (not shown) indicates that also the entire nonlinear dependence (fig. 3) of the synchronization time on $k$ and $q$ stays qualitatively the same for all these different systems.

Hence, in general small worlds do not synchronize fastest. This holds for various oscillator types, intrinsic dynamics and coupling schemes: phase oscillators coupled via phase differences, higher-dimensional periodic and chaotic systems coupled diffusively as well as neural circuits with inhibitory delayed pulse-coupling. In particular, small-world topologies are not at all special and may synchronize orders of magnitude slower than completely random networks. So generically the small-world regime can either exhibit slowest synchronization or just exhibit no extremal properties regarding synchronization times.

This phenomenon is rather unexpected given previous results on synchronization and small-world topology. For instance, the original work by Watts and Strogatz, as well as later studies $[7,9,10]$, indicate that small-world topologies support network synchronization, in particular they synchronize at weaker coupling strength than analogous, appropriately normalized globally coupled systems.

Apart from small-world properties, other topological features such as betweenness centrality, degree heterogeneity or hierarchical organization have been suggested to control whether or not a network actually synchronizes [26]. Our results now highlight that the speed of synchronization may vary several orders of magnitude, even if only the disorder in the topology changes. Synchronization speed thus serves as a key dynamic characteristic of oscillator networks, because even if a system synchronizes in principle, it might not in practice as the time scales involved may be much longer than those relevant to the system's function. For practical problems in realworld networks, such as preventing synchrony in neural circuits [4] or supporting synchrony in communication systems [3], it is thus essential to further systematically investigate how additional features, such as heterogeneities [27] or non-standard degree distributions [19], impact synchronization speed.

Supported by the BMBF under grant No. 01GQ0430, by a grant of the Max Planck Society to MT and by EPSRC grant No. EP/E501311/1 to SH and SG.

\section{REFERENCES}

[1] Pikovsky A., Rosenblum M. and Kurths J., Synchronization, A Universal Concept in Nonlinear Sciences (Cambridge University Press, Cambridge, UK) 2001. 
[2] Arenas A. et al., Phys. Rep., 469 (2008) 93.

[3] Kanter I., Kinzel W. and Kanter E., Europhys. Lett., 57 (2002) 141.

[4] Maistrenko Yu. et al., Phys. Rev. Lett., 93 (2004) 084102.

[5] Netoff T. et al., J. Neurosci., 24 (2004) 8075.

[6] Strogatz S., Nature, 410 (2001) 268.

[7] Nishikawa T. et al., Phys. Rev. Lett., 91 (2003) 014101.

[8] Pecora L. and Carroll T., Phys. Rev. Lett., 80 (1998) 2109.

[9] Watts D. and Strogatz S., Nature, 393 (1998) 440.

[10] Barahona M. and Pecora L. M., Phys. Rev. Lett., 89 (2002) 054101.

[11] Roxin A., Riecke H. and Solla S., Phys. Rev. Lett., 92 (2004) 198101.

[12] Zumdieck A. et al., Phys. Rev. Lett., 93 (2004) 244103.

[13] Zillmer R. et al., Phys. Rev. E, 76 (2007) 046102.

[14] Jahnke S., Memmesheimer R.-M. and Timme M., Phys. Rev. Lett., 100 (2008) 048102.

[15] Zillmer R., Brunel N. and Hansel D., Phys. Rev. E., 79 (2009) 031909.
[16] Timme M., Wolf F. and Geisel T., Phys. Rev. Lett., 92 (2004) 074101.

[17] Timme M., Geisel T. and Wolf F., Chaos, 16 (2006) 015108.

[18] QI G. X. et al., Phys. Rev. E, 77 (2008) 056205.

[19] QI G. X. et al., EPL, 82 (2008) 38003.

[20] Timme M., Europhys. Lett., 76 (2006) 367.

[21] Acebron J. et al., Rev. Mod. Phys., 77 (2005) 137.

[22] Denker M., Timme M., Diesmann M., Wolf F. and Geisel T., Phys. Rev. Lett., 92 (2004) 074103.

[23] Fagiolo G., Phys. Rev. E, 76 (2007) 026107.

[24] Almendral J. A. and Diaz-Guilera A., New J. Phys., 9 (2007) 1211.

[25] Mirollo R. and Strogatz S., SIAM J. Appl. Math., 50 (1990) 366.

[26] Motter A., Zhou C. and Kurths J., Phys. Rev. E, 71 (2005) 016116; GoH K. et al., Phys. Rev. Lett., 96 (2006) 018701; Lee S., Kim P. and Jeong H., Phys. Rev. E, 73 (2006) 016102; Arenas A., Diaz-Guilera A. and Perez-Vicente C. J., Phys. Rev. Lett., 96 (2006) 114102.

[27] Zhou C. and Kurths J., Chaos, 16 (2006) 015104. 